\title{
SUFFICIENT CONDITION FOR A MINIMUM WITH RESPECT TO
}

\section{ONE-SIDED VARIATIONS *}

BY

\section{GILBERT AMES BLISS}

In a problem of the calculus of variations with fixed endpoints, the curves along which the integral is taken may be free to traverse any portion of the plane, or they may be restricted to lie in a given region $R$. In the latter case it sometimes happens that although no solution can be found which lies entirely within the region, yet one exists consisting partly of interior arcs and partly of ares of the boundary. In the neighborhood of the boundary ares the only variations considered must be of course interior to the region, and therefore lie on one side only of the curve whose minimizing properties are to be investigated.

The necessary conditions which such a combination of interior curve and boundary must satisfy in order to minimize the given integral have already been derived. It is proposed in the following paper to prove that these conditions with slight modification are also sufficient: first, when the integral has the form

$$
I=\int f\left(x, y, y^{\prime}\right) d x ;
$$

and second, when the integral is

taken along a curve

in parameter representation.

$$
I=\int F\left(x, y, x^{\prime}, y^{\prime}\right) d t,
$$

$$
x=\mu(t), y=\nu(t)
$$

Part I. The problem for $I=\int f\left(x, y, y^{\prime}\right) d x$.

$\S 1$. The necessary conditions.

Consider an integral in the form (2) for which the function $f$ is of class $C^{\prime \prime \prime}, \dagger$ and $\partial^{\prime} f / \partial y^{\prime 2}>0, \ddagger$ in a region including in its interior all $\left(x, y, y^{\prime}\right)$ points which satisfy

* Presented to the Society (Chicago), April 2, 1904. Received for publication June 25, 1904.

† A function is said to be of class $C^{(i)}$ when it is continuous and has continuous derivatives up to and including those of order $i$. It is of class $D^{(i)}$ when the derivatives mentioned have only a finite number of discontinuities (compare BoLzA, Lectures on the Calculus of Variatione, The University of Chicago Press, Chicago, 1904, p. 7). Similarly a curve is of class $C^{(i)}$ or $D^{(i)}$ if the functions which represent it have the corresponding properties.

$\ddagger$ Problems in which $f$ has this property are called regular. 


$$
R^{\prime}: \quad\left\{\begin{array}{l}
(x, y) \text { in a region } R \text { of the } x y \text { plane } \\
\left|y^{\prime}\right| \leqq L
\end{array}\right.
$$

where $L$ is a positive constant. At least part of the boundary of $R$ is composed of a curve of class $D^{\prime \prime}$.

Consider a curve $E$ joining two given points $a$ and $b$ interior to $R$, and consisting for convenience of only three pieces $E_{1}, B, E_{2}$, each of class $C^{\prime \prime}$; the pieces $E_{1}$ and $E_{2}$ are interior to $R$ except at the points $c$ and $d$ where they

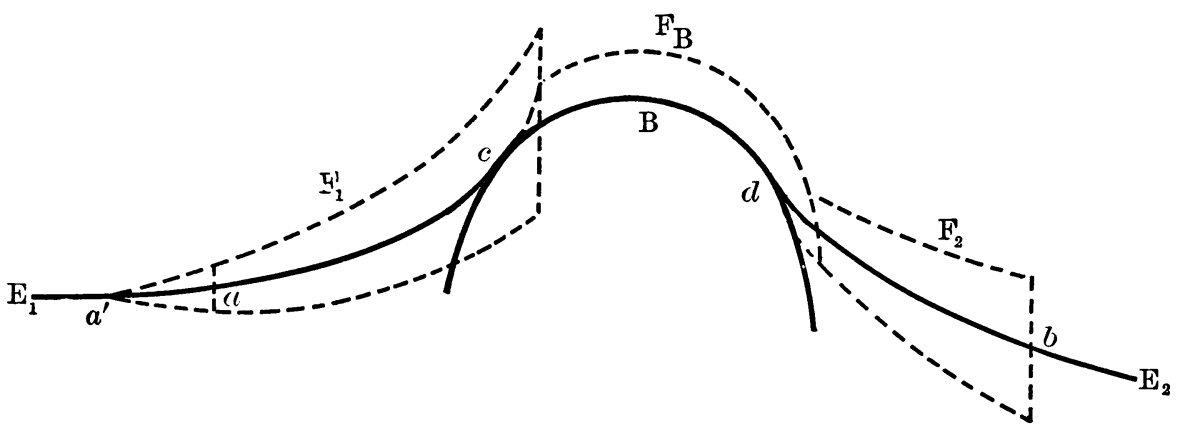

FiG. 1.

adjoin the arc $B$ of the boundary of $R$, as in Fig. 1. If this curve is to minimize $I$ the following necessary conditions must be satisfied :

I) $E_{1}$ and $E_{2}$ must be extremals, i. e. they must satisfy Euler's equation,

$$
f_{y}-\frac{d}{d x} f_{y^{\prime}}=0 ;^{*}
$$

II) No conjugate point to $a$ lies between $a$ and $c$ on $E_{1}$, and no conjugate point to $d$ lies between $d$ and $b$ on $E_{2} ; \dagger$

III) At $c$ and $d, E_{1}$ and $E_{2}$ are respectively tangent to $B ; \pm$

IV) Along $B$,

$$
f_{y}-\frac{d}{d x} f_{y^{\prime}} \leqq 0 . \S
$$

\footnotetext{
* See for example, Osgood, Sufficient Conditions in the Calculus of Variations, Annals of Mathematics, 2d Ser., vol. 2 (1900), p. 108 ; also BolzA, loc. cit., p. 22. The literal subscripts $x, y, y^{\prime}$ denote partial differentiation.

† BoLZA, loc. cit., pp. 63, 67.

$\ddagger$ BolzA, loc. cit., p. 42 footnote. The usual form of this condition is

$E\left(x, y, y^{\prime}, \beta^{\prime}\right)=f\left(x, y, \beta^{\prime}\right)-f\left(x, y, y^{\prime}\right)-\left(\beta^{\prime}-y^{\prime}\right) f_{y^{\prime}}\left(x, y, y^{\prime}\right)=0$ at $c$ and $d$, where $\beta^{\prime}$ and $y^{\prime}$ refer to the curves $B$ and the extremals $E_{1}, E_{2}$ respectively. But for a regular problem it follows from Taylor's formula that $E$ can vanish only when $y^{\prime}=\beta^{\prime}$.

$\S$ BoLzA, loc. cit., p. 43.
} 
It has been tacitly assumed that the region $R$ lies above the curve $B$. Otherwise condition IV would read $\geqq 0$.

If the conditions II and IV are amended to read

$\left.\mathrm{II}^{\prime}\right)$ No conjugate point to $a$ lies between $a$ and $c$ or at $c$ on $E_{1}$, and no conjugate point to $d$ lies between $d$ and $b$ or at $b$ on $E_{2}$;

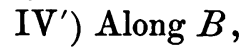

$$
f_{y}-\frac{d}{d x} f_{y^{\prime}}>0
$$

then I, II', III, IV' are sufficient conditions for a minimum. The proof of the last statement is the object of the present paper.

\section{§2. Existence theorems for Euler's equation.}

The differential equation (3), discovered by Euler, which characterizes the extremals for a problem of the calculus of variations of the form stated in $\S 1$, is of the second order. Since $f_{y^{\prime} y^{\prime}}>0$, it can be solved for $y^{\prime \prime}$ so that

$$
y^{\prime \prime}=e\left(x, y, y^{\prime}\right),
$$

where the function $e$ is of class $C^{\prime}$ in the region including $R^{\prime}$ in which the properties of $f$ were supposed to hold. In the sequel two existence theorems for this equation will be used, which are stated here without proof.*

The essence of the first theorem is that if one solution of equation (4) exists in the region $R^{\prime}$ through an initial point and direction $\left(\xi_{0}, \eta_{0}, \eta_{0}^{\prime}\right)$, it can always be surrounded by an infinity of other solutions all defined on the same interval and through neighboring initial elements $\left(\xi, \eta, \eta^{\prime}\right)$. In precise language, if

$$
y=\Phi(x)
$$

is a solution of equation (4) of class $C^{\prime \prime}$ on an interval $\left[\xi_{0} \xi_{1}\right]$, with initial values $\left(\xi_{0}, \eta_{0}, \eta_{0}^{\prime}\right)$, and entirely interior to $R^{\prime}$, then a function

$$
y=\phi\left(x, \xi, \eta, \eta^{\prime}\right)
$$

and a positive constant $\rho$ exist with the following properties :

1) The function $\phi$ and its $x$-derivatives $\phi^{\prime}, \phi^{\prime \prime}$ are of class $C^{\prime}$ for all values of $x, \xi, \eta, \eta^{\prime}$ satisfying the inequalities,

$$
\left|\xi-\xi_{0}\right| \leqq \rho,\left|\eta-\eta_{0}\right| \leqq \rho,\left|\eta^{\prime}-\eta_{0}^{\prime}\right| \leqq \rho, \xi_{0}-\rho \leqq x \leqq \xi_{1}+\rho ;
$$

2) The curve $y=\phi$ solves equation (4) and takes the initial element $\left(\xi, \eta, \eta^{\prime}\right)$ at $x=\xi$;

* See Picard, Traité d'Analyse, vol. 2, chap. 11; Peano, Atti di Torino, vol. 33 (1897), p. 9 ; and the references in the Encyclopüdie der Mathematischen Wissenschaften, IIA 4a, pp. 195, 200 . 
3) No other curve of class $C^{\prime \prime}$ on the interval $\left[\xi_{0}-\rho, \xi_{0}+\rho\right]$ has the properties 2).

On account of 3 ) it is seen that the curve (5) is represented by (6) when $\left(\xi, \eta, \eta^{\prime}\right)=\left(\xi_{0}, \eta_{0}, \eta_{0}^{\prime}\right)$.

Let $\mathrm{P}^{\prime}$ denote any perfect region of points $\left(\xi, \eta, \eta^{\prime}\right)$ in $R^{\prime}$. The second theorem states that one and only one solution of equation (4) exists through each element $\left(\xi, \eta, \eta^{\prime}\right)$ in $\mathbf{P}^{\prime}$, and gives a minimum interval in which such a solution is surely defined: i. e., there always exists corresponding to $\mathrm{P}^{\prime}$ a function

$$
y=\phi\left(x, \xi, \eta, \eta^{\prime}\right)
$$

and a positive constant $\rho$ with the properties:

1) The function $\phi$ and its $x$-derivatives $\phi^{\prime}, \phi^{\prime \prime}$ are of class $C^{\prime}$ in the region defined by the conditions,

$$
\left(\xi, \eta, \eta^{\prime}\right) \text { in } \mathrm{P}^{\prime}, \quad \xi \leqq x \leqq \xi+\rho ;
$$

2) The curve $y=\phi$ is a solution of equation (4) with the initial element $\left(\xi, \eta, \eta^{\prime}\right)$ when $x=\xi$;

3) No other curve of class $C^{\prime \prime}$ on $[\xi, \xi+\rho]$ has the properties 2).

\section{§ 3. The field.}

Suppose that the four conditions I, II', III, IV' of $\S 1$ are satisfied by the curve $E$. Then a set of extremals exist all passing through a point $a^{\prime}$ on the extremal $E_{1}$, which simply cover a portion of the plane, a so-called field, about the curve $E_{1}$. Let the element of $E_{1}$ at $a^{\prime}$ be $\xi, \eta, \bar{\eta}^{\prime}$ with $\xi<x_{a}$.

If $a^{\prime}$ is near enough to $a$ the former of the two existence theorems stated above, with $\xi_{0}, \eta_{0}, \eta_{0}^{\prime}$ replaced by $x_{a}, y_{a}, y_{a}^{\prime},{ }^{*}$ shows that there is a set of extremals

$$
y=\phi\left(x, \xi, \eta, \eta^{\prime}\right)=\psi_{1}\left(x, \eta^{\prime}\right),
$$

through the point $a^{\prime}$, and such that $\psi_{1}, \psi_{1}^{\prime}, \psi_{1}^{\prime \prime}$ are of class $C^{\prime}$ for

$$
\left|\eta^{\prime}-\bar{\eta}^{\prime}\right| \leqq \rho, \quad x_{a} \leqq x \leqq x_{c}+\delta,
$$

where $\rho$ and $\delta$ are two suitably chosen positive quantities.

As is well known the $x$-coördinate of the point conjugate to $(\xi, \eta)$ on one of the extremals (7), is defined by a zero of the derivative $\partial \phi / \partial \eta^{\prime} \cdot \dagger$ When $\left(\xi, \eta, \eta^{\prime}\right)=\left(x_{a}, y_{a}, y_{a}^{\prime}\right)$ this derivative determines the conjugate to $a$ on the extremal $E_{1}$ and on account of $\mathrm{IV}^{\prime}$ can not vanish anywhere except at $a$ on the arc $a c$.

* The literal subsoripts $a, b, c, d$ designate the coördinates of the corresponding points.

† Bolza, loc. cit., p. 62. 
It follows that, if $a^{\prime}, \delta, \rho$ are properly chosen, the derivative $\partial \psi_{1} / \partial \eta^{\prime}$ can not vanish anywhere in the region (8). Namely from $\S 1,2$ ),

and therefore

$$
\eta=\phi\left(\xi, \xi, \eta, \eta^{\prime}\right), \quad \eta^{\prime}=\phi^{\prime}\left(\xi, \xi, \eta, \eta^{\prime}\right),
$$

$$
0=\frac{\partial \bar{\phi}\left(\xi, \xi, \eta, \eta^{\prime}\right)}{\partial \eta^{\prime}}, \quad 1=\frac{\partial \phi^{\prime}\left(\xi, \xi, \eta, \eta^{\prime}\right)}{\partial \eta^{\prime}},
$$

in particular when $\left(\zeta, \eta, \eta^{\prime}\right)=\left(x_{a}, y_{a}, y_{a}^{\prime}\right)$. Hence on account of the continuity properties of $\phi$, an interval $\left[x_{a}-h, x_{a}+h\right]$ can be found such that the derivative $\partial \phi^{\prime} / \partial \eta^{\prime}$ is different from zero for all arguments $\left(x, \xi, \eta, \eta^{\prime}\right)$ for which $x$ is in the interval, $a^{\prime}$ on the corresponding arc of $E_{1}$, and $\eta^{\prime}$ the direction of $E_{1}$ at $a^{\prime}$. That is, $\partial \phi\left(x, \xi, \eta, \eta^{\prime}\right) / \partial \eta^{\prime}$ vanishes only at $x=\xi$ in the interval $\left[x_{a}-h, x_{a}+h\right]$. Let now $a^{\prime}$ approach $a$ on $E_{1}$. Then it must be possible to take $a^{\prime}$ so near to $a$, and a constant $\delta$ so small that the latter derivative is different from zero for all values of $x$ in $\left[x_{a}+h, x_{c}+\delta\right]$. Otherwise on account of continuity considerations $\partial \phi\left(x, x_{a}, y_{a}, y_{a}^{\prime}\right) / \partial y_{a}^{\prime}$ would vanish and the point $a$ would have a conjugate on the arc $a c$, which is contrary to hypothesis. But since $\partial \phi\left(x, \xi, \eta, \eta^{\prime}\right) / \partial \eta^{\prime}$ is different from zero when $\eta^{\prime}$ is the direction of $E_{1}$ at $a^{\prime}$, and $x$ satisfies the second inequality (8), it must also be different from zero for all points satisfying (8) when $\rho$ is properly restricted.

The field is the region $F_{1}$ of the $x y$-plane bounded by the four curve pieces which correspond to the boundary of the region (8) (see Fig. 1). That these curves are non-intersecting and divide the plane into an interior and an exterior, follows because the derivative $\partial \psi_{1} / \partial \eta^{\prime}$ is never zero, and consequently on any ordinate $\psi_{1}$ increases (decreases) from $\psi\left(x, \bar{\eta}^{\prime}-\rho\right)$ to $\psi\left(x, \bar{\eta}^{\prime}+\rho\right)$ as $\eta^{\prime}$ increases from $\bar{\eta}^{\prime}-\rho$ to $\bar{\eta}^{\prime}+\rho$. It is seen from this also that through each point of $F_{1}$, there passes one and but one extremal of the set (7). The function

$$
\eta^{\prime}=\eta^{\prime}(x, y)
$$

so defined in the region $F_{1}$ is of class $C^{\prime}$, since in the vicinity of any point of $F_{1}$ it is the solution of the equation

$$
y=\psi_{1}\left(x, \eta^{\prime}\right),
$$

which has continuous partial derivatives of the first order.*

In a manner somewhat similar a field $F_{B}$ can be constructed above the curve $B$ by means of a set of extremals each tangent to $B$. Let the equation of this are of the boundary be

$$
y=\beta(x) .
$$

* For the theorems on implicit functions see Jordax, Cours d'Analyse, vol. 1, p. 80. 
By the second existence theorem, with the totality of points and directions of $B$ as the region $\mathrm{P}^{\prime}$, there is a set of extremals

$$
y=\phi\left[x, \xi, \beta(\xi), \beta^{\prime}(\xi)\right]=\psi(x, \xi),
$$

each one of which is tangent to the curve $B$ at the point $[\xi, \beta(\xi)]$, and such that $\psi, \psi^{\prime}, \psi^{\prime \prime}$ are of class $C^{\prime}$ for

$$
x_{c} \leqq \xi \leqq x_{d}+\epsilon, \quad \xi \leqq x \leqq \xi+\rho,
$$

provided that $\epsilon, \rho$ are properly chosen. The derivative $\partial \psi / \partial \xi$ is a solution of the linear differential equation

$$
u^{\prime \prime}=u e_{y}\left(x, \psi, \psi^{\prime}\right)+u^{\prime} e_{y^{\prime}}\left(x, \psi, \psi^{\prime}\right),
$$

as is easily seen by substituting the expression (9) in equation (4) and differentiating for $\xi$. From the identities

it appears that

$$
\beta(\xi)=\psi(\xi, \xi), \quad \beta^{\prime}(\xi)=\psi^{\prime}(\xi, \xi),
$$

$$
\begin{aligned}
& \left.\frac{\partial \psi}{\partial \xi}\right|^{x=\xi}=\beta^{\prime}(\xi)-\psi^{\prime}(\xi, \xi)=0, \\
& \left.\frac{\partial \psi^{\prime}}{\partial \xi}\right|^{x=\xi}=\beta^{\prime \prime}(\xi)-\psi^{\prime \prime}(\xi, \xi)<0,
\end{aligned}
$$

the latter on account of the condition IV'. According to a theorem of PICARD, the positive constant $\rho$ can be so restricted independently of $\xi$ that the only integral of equation (11) which vanishes twice on the interval $[\xi, \xi+\rho]$ is $u \equiv 0 . \dagger$ Equations (12) show therefore that $\partial \psi / \partial \xi$ is not identically zero, but less than zero when $\xi<x \leqq \xi+\rho$.

The field $F_{B}$ is the interior of the region bounded by the curves in the $x y$ plane which correspond to the boundaries of the region (10) (see Fig. 1). From the properties of the derivative $\partial \psi / \partial \xi$ it can be shown as before that these curves do not intersect, and that the region which they bound is simply covered by the extremals of the set (9). The single-valued function

$$
\xi=\xi(x, y)
$$

which defines the extremal through a given point, is continuous over the whole

$\dagger$ PICARD, Traite d'Analyse, vol. 3, p. $94 \mathrm{ff}$. If $\alpha$ and $\beta$ are the maxima of $\left|e_{y}\right|$ and $\left|e_{y^{\prime}}\right|$ in the region (10), then $\rho$ may be any positive quantity satisfying

$$
\alpha \frac{\rho^{2}}{8}+\beta_{2}^{\rho}<1
$$

since equation (11) is linear (loc. cit., pp. 97, 100). 
region $F_{B}$, and has continuous first partial derivatives everywhere except on the curve $B$. These results follow easily from the theorems on implicit functions when $(x, y)$ is not on the curve $B$, since the function $\xi(x, y)$ is the solution of

$$
y=\psi(x, \xi) .
$$

As $(x, y)$ approaches a point $\left(x_{1}, y_{1}\right)$ on the curve $B$,

$$
\lim \xi(x, y)=x_{1},
$$

as is readily proved by supposing some other limit, and showing that the corresponding extremal cannot pass through $\left(x_{1}, y_{1}\right)$. The partial derivatives are in general infinite, for

and $\psi_{\xi}$ vanishes along $B$.

$$
\begin{aligned}
& 0=\psi_{x}+\psi_{\xi} \xi_{x}, \\
& 1=\psi_{\xi} \xi_{y},
\end{aligned}
$$

The first existence theorem of $\S 1$ with $\xi_{0}, \eta_{0}, \eta_{0}^{\prime}$ replaced by $x_{d}, y_{d}, y_{d}^{\prime}$ shows that the extremals of the set (9) for which

$$
x_{d}-\epsilon \leqq \xi \leqq x_{d}+\epsilon,
$$

can be extended over the interval

$$
\xi+\rho \leqq x \leqq x_{b},
$$

so as to form a field about the curve $E_{2}$. The derivative $\partial \psi / \partial \xi$ cannot vanish for any values of $x, \xi$ satisfying (14) and (15), if $\epsilon$ is suffificiently small,

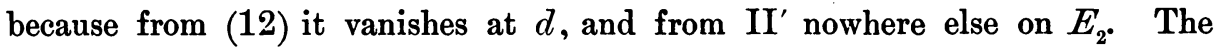
curves corresponding to the boundary of the region defined by (14) and (15), bound a field $F_{2}$ in the $x y$-plane about the extremal $E_{2}$, similar to $F_{1}$ about $E_{1}$.

The three regions $F_{1}, F_{B}, F_{2}$ form a simply connected plane region $F$. For part of the boundary of $F_{B}$ is in $F_{1}$, and $F_{B}$ and $F_{2}$ have a strip of boundary in common.

The result is then that in the neighborhood of the curve $E$ a simply connected plane region $F$ can be found such that each point of $F$ can be joi. ed with the point $a^{\prime}$ by a curve $C$ consisting either of an extremal which does ' ot intersect $E$, or else of an arc of $E$ plus an arc of an extremal which is ta gent to $E$ (see Fig. 1). There is only one such curve $C$ connecting $a^{\prime}$ with any point of $F$.

\section{§4. The Weierstrass construction in the field $F$.}

With the results of the last section in mind it can be shown by a method of WeIERSTRAss* that the curve $E$ minimizes the integral $I$ with respect to all

* See BolzA, loc. cit., pp. 84-91 ; also Osgood, loc. cit., p. 115. 
variations $\mathrm{V}$ of class $D^{\prime}$ which lie in $F$, join $a$ and $b$, and do not cross the curve $B$. This is effected by means of a function $W$ defined as follows: Let $p(\bar{x}, \bar{y})$ be a point which is free to vary along $V$, then

$$
W(\bar{x})=I_{C}\left(a^{\prime} p\right)+I_{V}(p b),
$$

where $C$ is the curve described in $\S 3$ joining the point $a^{\prime}$ with $p, I_{C}\left(a^{\prime} p\right)$ represents the integral $I$ taken along the curve $C$ from $a^{\prime}$ to $p$, and $I_{r}(p b)$ has an

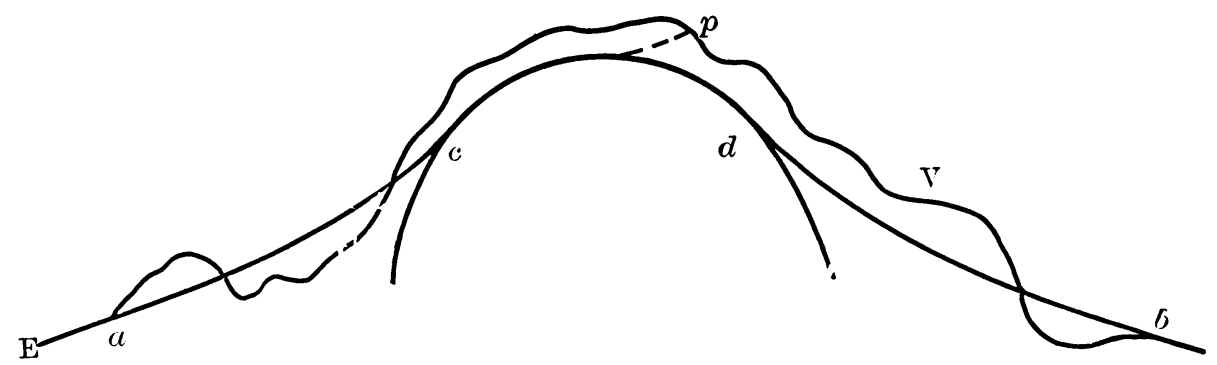

FIG. 2.

analogous meaning (see Fig. 2) with respect to the variation V. The expression whose sign is to be determined is

$$
\Delta I=I_{v}(a b)-I_{E}(a b)=W\left(x_{a}\right)-W\left(x_{b}\right),
$$

and the sign consequently depends upon the behavior of the function $W(\bar{x})$ on the interval $\left[x_{a}, x_{b}\right]$.

The explicit expression for the function $W(\bar{x})$ is different according as the point $p$ lies in the field $F_{1}$ or not. It will be sufficient if its properties are discussed for a point in one of the other fields $F_{B}$ or $F_{2}$. In this case

$$
\begin{aligned}
W(\bar{x})=I_{E_{1}}\left(a^{\prime} c\right)+\int_{x_{c}}^{\xi} f\left(x, \beta, \beta^{\prime}\right) d x & \\
& +\int_{\xi}^{\bar{x}} f^{\prime}\left(x, \psi, \psi^{\prime}\right) d x+\int_{\bar{x}}^{x_{b}} f\left(x, g, g^{\prime}\right) d x,
\end{aligned}
$$

where $y=g(x)$ is the equation of $\mathrm{V}$, and the function $\xi$ in the limit of the integral and in $\psi$ has the arguments $\bar{x}, g(\bar{x})$ instead of $x$ and $y$ (see the figure). It follows at once from the properties of the function $\xi$ that $W$ is a continuous function of $\bar{x}$. When $p$ is interior to one of the fields $F_{B}$ or $F_{2}$, the derivative of $W$ is

$$
\begin{aligned}
\frac{d W}{d \bar{x}}=[ & \left.f\left(x, \beta, \beta^{\prime}\right)-f\left(x, \psi, \psi^{\prime}\right)\right]^{x=\xi} \frac{d \xi}{d \bar{x}} \\
& +\frac{d \xi}{d \bar{x}} \int_{\xi}^{\bar{x}}\left\{f_{\psi}^{\partial \psi^{\prime}}+f_{\psi^{\prime}} \frac{\partial \psi^{\prime}}{\partial \xi}\right\} d x+\left[f\left(x, \psi, \psi^{\prime}\right)-f\left(x, g, g^{\prime}\right)\right]^{r=\bar{x}} .
\end{aligned}
$$


Since $B$ and the extremals (9) are tangent at $x=\xi$, this becomes by partial integration and use of the equation (3) which is satisfied by the extremals (9);

But

$$
\frac{d W}{d \bar{x}}=-f\left(x, g, g^{\prime}\right)+f\left(x, \psi, \psi^{\prime}\right)+\left.\frac{d \xi}{d \bar{x}} \frac{\partial \psi}{\partial \xi} f_{\psi^{\prime}}\left(x, \psi, \psi^{\prime}\right)\right|^{x=\bar{x}} .
$$

$$
g(x) \equiv \psi(x, \xi[x, g(x)]),
$$

since $\xi(x, y)$ is the solution of equation (13), and therefore

$$
\begin{gathered}
g^{\prime}-\psi^{\prime}=\frac{\partial \psi}{\partial \xi} \frac{d \xi}{d x}, \\
\frac{d W}{d \bar{x}}=-\left.E\left(x, y, \psi, \psi^{\prime}, g, g^{\prime}\right)\right|^{x=\bar{x}},
\end{gathered}
$$

where$$
E=f\left(x, g, g^{\prime}\right)-f\left(x, \psi, \psi^{\prime}\right)-\left(g^{\prime}-\psi^{\prime}\right) f_{\psi^{\prime}}\left(x, \psi, \psi^{\prime}\right) .
$$

The function $E$ is always positive or zero and vanishes only when $g^{\prime}=\psi^{\prime}$. For by TAYLOR's formula

$$
E=\frac{1}{2}\left(g^{\prime}-\psi^{\prime}\right)^{2} f_{\psi^{\prime} \psi^{\prime}}\left[x, \psi, \psi^{\prime}+\theta\left(g^{\prime}-\psi^{\prime}\right)\right],
$$

and for a regular problem $f_{\psi^{\prime} \psi^{\prime}}$ is always $>0$.

For a point $p$ in the field $F_{1}$, the derivative of $W$ is the same except that $\psi$ must be replaced by $\psi_{1}$ : The only points remaining to be considered are points where the curve $V$ coincides with the part $\bar{E}_{1}$ of $E_{1}$ which is common to $F_{1}$ and $F_{B}$, or with the boundary $B$. At an interior point of an interval common with one of these curves, it follows readily geometrically that $W$ has a derivative which is zero, for as $(\bar{x}, \bar{y})$ varies along the interval $W$ remains constant. At the endpoints of such an interval, however, the derivative does not in general exist.

To avoid unnecessary complication it will be assumed that $V$ has only a finite number of intervals in common with $\bar{E}_{1}$ or $B$. Then on the interval $\left[x_{a}, x_{b}\right]$ the function $W$ is continuous and has a derivative continuous and $\leqq 0$ except in a finite number of points. The expression $\Delta I$ will therefore be positive unless $d W / d \bar{x}$ is identically zero in $\left[x_{a}, x_{b}\right]$. This could happen, however, only if $g^{\prime}$ were equal to $\psi^{\prime}$ or $\psi_{1}^{\prime}$ at every point of $V$. In that case $V$ would be of class $C^{\prime}$ since, for example, in $F_{B}$ or $F_{2}$ the equation

$$
g^{\prime}(x)=\psi^{\prime}[x, \xi(x), g(x)]
$$

implies the continuity of $g^{\prime}$. Furthermore $V$ would be an extremal whenever it did not coincide with $E$. For from (16) $\xi$ must be constant along such an arc. 
The only curve in the field with these properties through the point $b$ is $E$ itself, with which therefore $V$ would have to coincide.

The result is then that the curve $E$ gives to the integral $I$ a smaller value than that given by any other curve of class $D^{\prime}$ in the field $F$ joining the points $a$ and $b$.

A similar theorem holds true when the curve $E$ consists of more than three pieces. Arcs of extremals will then alternate with ares of the boundary of the region $R$, and a field can be constructed in the neighborhood of each in the manner described above. The plane region covered by the totality of these fields has the properties of the field $F$ used in the simpler case.

\section{Part II. The problem in Parameter Representation.}

\section{$\S 1$. The necessary condition.}

The function $F$ in the integral (2) is assumed to have the following properties in a region of points $\left(x, y, x^{\prime}, y^{\prime}\right)$ including in its interior

$$
R^{\prime}:\left\{\begin{array}{l}
(x, y) \text { in a given region } R \text { of the } x y \text { plane, } \\
\left(x^{\prime}, y^{\prime}\right) \text { any values not both zero: }
\end{array}\right.
$$

1) It is of class $C^{\prime \prime \prime}$;

2) For all values of $\kappa$ greater than zero,

$$
\begin{gathered}
F\left(x, y, \kappa x^{\prime}, \kappa y^{\prime}\right)=\kappa F\left(x, y, x^{\prime}, y^{\prime}\right) ; \\
F_{1}=\frac{1}{y^{\prime 2}} F_{x^{\prime} x^{\prime}}=-\frac{1}{x^{\prime} y^{\prime}} F_{x^{\prime} y^{\prime}}^{\prime}=\frac{1}{x^{\prime 2}} F_{y^{\prime} y^{\prime}}>0 . *
\end{gathered}
$$

The region $R$ in which all the curves considered are supposed to lie has a curve of class $D^{\text {iv }}$ as its boundary. As before a curve $E$ will be considered consisting of three pieces $E_{1}, B, E_{2}$. The method used in constructing the field makes it necessary to assume that these pieces are of class $C^{\mathrm{iv}}$.

The necessary and the sufficient conditions that $E$ shall minimize the integral read the same as before except that the Euler expression must be replaced by

$$
G=F_{x y^{\prime}}-F_{x^{\prime} y}+F_{1}\left(x^{\prime} y^{\prime \prime}-x^{\prime \prime} y^{\prime}\right) \dagger
$$

\footnotetext{
* For these assumptions on $F$ see BoLzA, loc. cit., p. 117. The function $F_{1}$ plays a role analogous to that of $f_{y^{\prime} y^{\prime}}$ in the previous case.

† It has been tacitly assumed that the parameter representation on the boundary of $R$ has been so chosen that the interior of $R$ lies to the left as one faces the direction in which the parameter increases. See BolzA, loc. cit., pp. 123, 148.
} 


\section{§2. An auxiliary theorem.}

The existence of a field about one of the pieces of $E$ follows from the results in the preceding sections provided that the arc of the piece in question and the distances along its normals are taken as new coördinates. The use of these coördinates in a region adjoining the curve is justified by the following theorem : The normals to a curve of class $C^{(i)}(i>1)$ simply cover a portion of the plane surrounding the curve. In this plane region the arc of the given curve and the distances along the normals can be taken as new coördinates and the transformation so defined will be of class $C^{(i-1)}$.*

Let the equations of the curve be

$$
x=g(s), \quad y=h(s),
$$

where $s$ is the length of the curve measured from some initial point, and let $s_{0}, s_{1}$ correspond to the ends of the arc under consideration. Suppose furthermore that the curve has no singular points on the are $\left[s_{0}, s_{1}\right]$, i. e., $g^{\prime 2}+{h^{\prime}}^{2} \neq 0$. The normal at any point has the equations

$$
x=g-h^{\prime} t, \quad y=h+g^{\prime} t,
$$

with $t$ as the parameter. It is possible to take a positive constant $\kappa$ so small that any two pairs of values $(s, t)$ which satisfy

$$
s_{0} \leqq s \leqq s_{1}, \quad|t| \leqq \kappa
$$

will define different $x y$ points. For suppose that this were not so. Then for each term of an arbitrarily chosen series of positive constants $\kappa_{i}(i=1,2, \ldots)$ with limit zero, there would exist two points, $P_{i}\left(s_{i}, t_{i}\right)$ and $P_{i}^{\prime}\left(s_{i}^{\prime}, t_{i}^{\prime}\right)$, lying in the region (19) defined by $\kappa_{i}$, and such that the corresponding points $\left(x_{i}, y_{i}\right)$ and $\left(x_{i}^{\prime}, y_{i}^{\prime}\right)$ defined by equations (18) would be identical. The points $P_{i}, P_{i}^{\prime}$ approach two limiting points $(s, 0)$ and $\left(s^{\prime}, 0\right)$ as $i$ increases, and these must also define identical $x y$ points. But this is impossible unless $s=s^{\prime}$, because the curve is supposed not to intersect itself. The two series of points have therefore the same limit point $(s, 0)$. This leads, however, to a contradiction. For the Jacobian of equations (18) with respect to $s$ and $t$ is

$$
\left(g^{\prime 2}+h^{\prime 2}\right) \neq 0
$$

at any point $(s, 0)$, and by the theorems on implicit functions there is always a region of $(s, t)$ points surrounding $(s, 0)$ such that any two points of the region define different $x y$ points by means of the equations (8).

* I. e., the functions defining the transformation and their inverses will be of class $C^{(i-1)}$. The method used in the proof of this theorem is similar to the one used by BouzA in the construction of a field, loc. cit., § 34 . 
Suppose then a $\kappa$ selected so that two different points of the region (19) define different $x y$ points, and so that the determinant of equations (18),

$$
\left|\begin{array}{ll}
x_{s} & y_{s} \\
x_{t} & y_{t}
\end{array}\right|,
$$

is different from zero in the whole region. The four curve pieces defined by the points $(s, t)$ on the boundary of (19) form a continuous non-intersecting curve in the $x y$-plane. By a theorem of Schoenflies * all the $x y$ points corresponding to the region (19) lie in the interior $\bar{R}$ of this curve or on the curve itself; and conversely, to each point of $\bar{R}$ there corresponds a point $(s, t)$ of (19). The equations (18) have therefore single valued solutions $s$ and $t$ in terms of $x$ and $y$ over the region $\bar{R}$, and by the theorems on implicit functions these solutions must be of class $C^{(i-1)}$.

\section{§ 3. Construction of the field.}

By means of the theorem just proved the problem of constructing a field adjoining one of the pieces of $E$ can be transformed to the $s t$-plane with $s$ as the independent variable. The integral of the transformed problem is

where

$$
I=\int f\left(s, t, \frac{d t}{d s}\right) d s
$$

$$
f=F\left(x(s, t), y(s, t), x_{s}+x_{t} \frac{d t}{d s}, y_{s}+y_{t} \frac{d t}{d s}\right) \text {. }
$$

The relations between the Euler equations and the functions $f_{t^{\prime} t^{\prime}}$ and $F_{1}$ are readily calculated and found to be

$$
\begin{aligned}
f_{t}-\frac{d}{d s} f_{t^{\prime}} & =\left|\begin{array}{ll}
x_{s} & y_{s} \\
x_{t} & y_{t}
\end{array}\right| G, \\
f_{t^{\prime} t^{\prime}} & =\left|\begin{array}{ll}
x_{s} & y_{s} \\
x_{t} & y_{t}
\end{array}\right|^{2} F_{1},
\end{aligned}
$$

and from (20) the determinant is positive. Consider for example the extremal $E_{1}$. It can be extended slightly at each end of the arc $a c$, and a region $\bar{R}_{1}$ which is pictured in a one to one way on a region $S_{1}$ in the st-plane, can be constructed as in the preceding section about the extended arc. It is seen that the initial assumptions and conditions I, III, $\mathrm{IV}^{\prime}$ of $\S 1$ for the function $f$ follow from those of part II, $\S 1$, for the function $F$. The region in

* See OSGOOD, Über einen Satz des Herrn Schoenflies aus der Theorie der Functionen zweier reellen Veränderlichen, Göttinger Nachrichten, 1900. 
which they hold consists of all points $\left(s, t, t^{\prime}=d t / d s\right)$ for which $(s, t)$ is in $S_{1}$ and $|d t / d s|$ bounded by any arbitrarily chosen positive constant $L$.

The Jacobi condition II' is also satisfied. For if

$$
x=\mu(s, a), \quad y=\nu(s, a)
$$

is any set of extremals containing $E_{1}$ in the $x y$-plane and corresponding to the set of extremals

$$
t=\phi(s, a)
$$

in the st-plane, the functional determinant is

$$
\left|\begin{array}{cc}
\mu_{s} & \mu_{a} \\
\nu_{s} & \nu_{a}
\end{array}\right|=\left|\begin{array}{ll}
x_{s} & x_{t} \\
y_{s} & y_{t}
\end{array}\right| \phi_{a}
$$

The determinant on the left cannot vanish twice on the curve $E_{1}$, for its zeros determine conjugate points. ${ }^{*}$ Consequently for any set (21) which contains the transform of $E_{1}^{\prime}$, the derivative $\phi_{a}$ cannot vanish twice on the segment of the $s$-axis which corresponds to the arc $a c$, and this segment in the st-plane cannot contain conjugate points.

It follows that in the st-plane a field can be constructed about the transform of $E_{1}$ with a set of extremals

$$
t=\phi_{1}\left(s_{1}, \sigma_{1}\right), \quad s_{a}-\delta \leqq s \leqq s_{c}+\delta, \quad\left|\sigma_{1}\right| \leqq \rho,
$$

where $s_{a}$ and $s_{c}$ are the lengths of ares of the curve $E$ measured from some fixed point to $a$ and $c$. In the $x y$-plane these take the form

$$
x=\mu_{1}\left(s_{1}, \sigma_{1}\right), \quad y=\nu_{1}\left(s_{1}, \sigma_{1}\right)
$$

and on account of the character of the transformation (18) simply cover as before a region $F_{1}$ surrounding $E_{1}$. Similarly a set of extremals exists in the st-plane

$$
t=\psi(s, \sigma), \quad s_{c} \leqq \sigma \leqq s_{d}+\epsilon, \quad \sigma \leqq s \leqq \sigma+\rho,
$$

tangent to the $s$ axis at $s=\sigma$, which in the $x y$-plane have the form

$$
x=\mu(s, \sigma), \quad y=\nu(s, \sigma),
$$

and sweep out a field $F_{B}$ adjoining the boundary $B$. By lengthening the arc $d b$ of $E_{2}$ and transforming again to the st-plane, a set of extremals

$$
x=\mu_{2}\left(s_{2}, \sigma_{2}\right), \quad y=\nu_{2}\left(s_{2}, \sigma_{2}\right)
$$

* See Bolza, loc. cit., p. 136. 
can be found, which are defined for

$$
\sigma_{2} \leqq s_{2} \leqq s_{b}+\rho, \quad s_{d}-\epsilon \leqq \sigma_{2} \leqq s_{d}+\epsilon,
$$

and are tangent to the curve $B$ for $s_{2}=\sigma_{2}$. These extremals coincide with those of the set (23) in the vicinity of the curve $B$, and simply cover a field about $E_{2}$.

The regions $F_{1}, F_{B}, F_{2}$ form a field $F$ about the curve $E$. Through every point of $F$ not on the curve $B$ there passes one extremal of the field. Each point of the field is joined to the point $a^{\prime}$ (see Fig. 2) by one and but one curve of class $C^{\prime}$ which consists of a finite number of arcs of $B$ and of extremals of the field tangent to $B$. In forming the field the part of $F_{1}$ which overlaps $F_{B}$ is to be neglected. The boundary $B_{1}$ between the two will then be part of the extremal $E_{1}$. Similarly $F_{B}$ and $F_{2}$ can be taken to have part of the curve (24) corresponding to $\sigma_{2}=s_{d}-\epsilon$ as boundary $B_{2}$. In the field $F_{1}$ the values $s_{1}$ and $\sigma_{1}$ defining the extremal through a point $(x, y)$ are single valued functions of $x$ and $y$, and of class $C^{\prime \prime}$ since they solve equations (22). The functions $s, \sigma$ and $s_{2}, \sigma_{2}$ have similar properties in $F_{B}$ and $F_{2}$, except on the curve $B$. For points on $B$ they are continuous but the derivatives are not finite.

\section{§4. The Weierstrass construction.}

If $V$ is a curve of class $D^{\prime}$ in the field $F$, with the equations

$$
x=\bar{x}(\tau), \quad y=\bar{y}(\tau),
$$

and joining $a\left(\tau=\tau_{0}\right)$ with $b\left(\tau=\tau_{1}\right)$, then a function $W$ can be defined geometrically as before along $V$. In the present case, however, $W$ is a function of $\tau$ instead of $\bar{x}$. At any point of $V$ which is not the endpoint of an interval coincident with $B_{1}, B_{2}$ or $B, W$ has the derivative

$$
\frac{d W}{d \tau}=-E\left(x, y, \mu^{\prime}, \nu^{\prime}, \bar{x}^{\prime}, \bar{y}^{\prime}\right)
$$

where primes denote differentiation with respect to $s$ or $\tau$, and

$$
E=F\left(x, y, \bar{x}^{\prime}, \bar{y}^{\prime}\right)-\bar{x}^{\prime} F_{x^{\prime}}\left(x, y, \mu^{\prime}, \nu^{\prime}\right)-\bar{y}^{\prime} F_{y^{\prime}}\left(x, y, \mu^{\prime}, \nu^{\prime}\right) .
$$

But, according to W EJERstrass, *

$$
E=(1-\cos l) \cdot F_{1}\left(x, y, \cos \left(\lambda+l_{1}\right), \sin \left(\lambda+l_{1}\right) \cdot \sqrt{\bar{x}^{\prime 2}+\bar{y}^{\prime 2}},\right.
$$
where

\footnotetext{
* Bolza, loc. cit., p. 140.
} 


$$
\begin{aligned}
\cos \lambda & =\frac{\mu^{\prime}}{\sqrt{\mu^{\prime 2}+\nu^{\prime 2}}}, & \sin \lambda & =\frac{\nu^{\prime}}{\sqrt{\mu^{\prime 2}+\nu^{\prime 2}}}, \\
\cos (\lambda+l) & =\frac{\bar{x}^{\prime}}{\sqrt{{\overline{x^{\prime 2}}}^{2}+\bar{y}^{\prime 2}}}, & \sin (\lambda+l) & =\frac{\bar{y}^{\prime}}{\sqrt{{\overline{x^{\prime}}}^{2}+\bar{y}^{\prime 2}}},
\end{aligned}
$$

and $l_{1}$ is a value between 0 and $l$. Consequently $E$ is always positive unless $l \equiv 0$ along $V$. If therefore $V$ has only a finite number of intervals in common with $B_{1}, B_{2}$ and $B$, and if $l \neq 0$ on $V$, then

is surely positive.

$$
\Delta I=W\left(\tau_{0}\right)-W\left(\tau_{1}\right)
$$

If $l \equiv 0$ along $V$, then $V$ and $E$ must coincide throughout. For the equations

$$
\frac{\bar{x}^{\prime}}{\sqrt{{x^{\prime 2}}^{2}+y^{\prime 2}}}=\frac{\mu^{\prime}}{\sqrt{{\mu^{\prime 2}}^{2}+\nu^{\prime 2}}}, \quad \frac{\bar{y}^{\prime}}{\sqrt{{x^{\prime 2}}^{2}+{y^{\prime 2}}^{2}}}=\frac{\nu^{\prime}}{\sqrt{\mu^{\prime 2}+\nu^{\prime 2}}},
$$

show that $V$ is of class $C^{\prime}$. In the vicinity of any point of $V$ not on $E$ it must coincide with the extremal of the field through that point. For from equations (23), for example, when $x$ and $y$ are replaced by the expressions (25),

$$
\begin{aligned}
& \bar{x}^{\prime}=\mu^{\prime} \frac{d s}{d \tau}+\mu_{\sigma} \frac{d \sigma}{d \tau}, \\
& \bar{y}^{\prime}=\nu^{\prime} \frac{d s}{d \tau}+\mu_{\sigma} \frac{d \sigma}{d \tau} .
\end{aligned}
$$

But from (26),

$$
\begin{aligned}
& 0=\mu^{\prime}\left(\frac{d s}{d \tau}-\frac{\sqrt{{\overline{x^{\prime}}}^{2}+\bar{y}^{\prime 2}}}{\sqrt{{\mu^{\prime 2}+\nu^{\prime 2}}^{2}}}\right)+\mu_{\sigma} \frac{d \sigma}{d \tau}, \\
& 0=\nu^{\prime}\left(\frac{d s}{d \tau}-\frac{\sqrt{{\overline{x^{\prime}}}^{2}+\bar{y}^{\prime 2}}}{\sqrt{{\mu^{\prime 2}+\nu^{\prime 2}}^{2}}}\right)+\nu_{\sigma} \frac{d \sigma}{d \tau},
\end{aligned}
$$

and these can only hold if $\sigma$ is constant as $\tau$ varies, and therefore $V$ an extremal. But $E$ is the only curve of the field with the properties just found and passing through the point $b$. It follows then that $V$ must be the curve $E$ itself.

It has been proved that for the problem in parameter representation the curve $E$ minimizes the integral $I$ with respect to all variations of class $D^{\prime}$ in the field $F$. 
The derivation of the necessary and of the sufficient conditions for the problem in parameter representation is of especial interest because it has been proved by Bolza, ${ }^{*}$ following a method suggested by HILBERT, $\dagger$ that there is certainly one curve in the region $R$ joining the points $a$ and $b$, which gives to the integral $I$ its minimum value, provided that proper assumptions are made upon the function $F$. In the simpler case when $I$ has the form (1) this is not always true. The necessary conditions given above characterize this solution of the problem, and as has been seen, when altered slightly, they are also sufficient for a minimum in a certain neighborhood of the curve.

The University of Chicago.

* Loc. cit., chap. vii, pp. 245-263.

$\dagger$ Lectures on the Calculus of Variations, Göttingen, Summer, 1900. 